VOL. 55 (1997) [321-325]

\title{
THE WEYL FUNCTIONAL CALCULUS AND TWO-BY-TWO SELFADJOINT MATRICES
}

\author{
WERNER J. RICKER
}

Let $D$ be a $(2 \times 2)$ matrix with distinct eigenvalues $\lambda_{1}$ and $\lambda_{2}$. There is a basic and well known functional equation which provides a formula for constructing the matrix $g(D)$, for any $\mathbb{C}$-valued function $g$ defined on a subset of $\mathbb{C}$ containing $\left\{\lambda_{1}, \lambda_{2}\right\}$, namely

$$
g \mapsto g(D)=\left(\lambda_{1}-\lambda_{2}\right)^{-1}\left\{g\left(\lambda_{1}\right) \cdot\left(D-\lambda_{2} I\right)-g\left(\lambda_{2}\right) \cdot\left(D-\lambda_{1} I\right)\right\} .
$$

This equation is used to give a direct and transparent proof of the following fact due to Anderson: A pair of $(2 \times 2)$ selfadjoint matrices $A_{1}$ and $A_{2}$ commute if and only if the Weyl functional calculus of the pair $\left(A_{1}, A_{2}\right)$, which is a matrix-valued distribution, has order zero (that is, is a measure).

Given two selfadjoint matrices in $\mathcal{H}=\mathbb{C}^{2}$, say $A_{1}, A_{2}$, the Weyl calculus for the pair $A=\left(A_{1}, A_{2}\right)$ is an $L(\mathcal{H})$-valued distribution which is a particular rule allowing the construction of certain functions of the pair $\left(A_{1}, A_{2}\right)$. For $\xi=\left(\xi_{1}, \xi_{2}\right) \in \mathbb{R}^{2}$, the matrix $\langle\xi, A\rangle=\xi_{1} A_{1}+\xi_{2} A_{2}$ is again selfadjoint and hence $\left\|e^{i\langle\xi, A\rangle}\right\|=1$. Let $\mathcal{S}\left(\mathbb{R}^{2}\right)$ denote the Schwartz space of $\mathbb{C}$-valued, rapidly decreasing functions on $\mathbb{R}^{2}$. More precisely then, the Weyl calculus for $A,[1,6,7]$, is the $L(\mathcal{H})$-valued distribution $T(A)$ defined by

$$
T(A) f=\frac{1}{2 \pi} \int_{\mathbb{R}^{2}} e^{i(\xi, A)} \hat{f}(\xi) d \xi, \quad f \in \mathcal{S}\left(\mathbb{R}^{2}\right)
$$

here $\hat{f}$ denotes the Fourier transform of $f$ and $L(\mathcal{H})$ is the space of all $(2 \times 2)$ matrices over $\mathbb{C}$. The following result connects an analytic property of $T(A)$ with a purely algebraic property of $A$.

Theorem 1. Given a pair $A=\left(A_{1}, A_{2}\right)$ of selfadjoint matrices in $\mathcal{H}=\mathbb{C}^{2}$ the following statements are equivalent.

(i) The matrices $A_{1}$ and $A_{2}$ commute.

(ii) The associated Weyl calculus $T(A): \mathcal{S}\left(\mathbb{R}^{2}\right) \rightarrow L(\mathcal{H})$ is a distribution of order zero.

Received 4th June, 1996

Copyright Clearance Centre, Inc. Serial-fee code: 0004-9729/97 \$A2.00+0.00. 
There are several proofs of this theorem in the literature. The first proof given of this result is due to Anderson, [2, Theorem 2], and applies in $\mathbb{C}^{m}$, not just $\mathbb{C}^{2}$. It is based on properties of the numerical range and the theory of multivariable differential calculus. A completely different proof (also applying in $\mathbb{C}^{m}$ ), which is based on certain

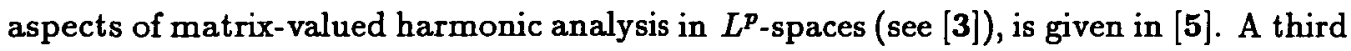
proof, specific to the case of $\mathbb{C}^{2}$, was given in [4]. This proof is essentially computational and is based on an elegant formula of Anderson, [1, Theorem 4.1], which expresses the Weyl calculus $T(J)$ of the triple $J=\left(J_{1}, J_{2}, J_{3}\right)$ whose entries are the classical spin $1 / 2$-matrices in $L\left(\mathbb{C}^{2}\right)$, in terms of an integral formula over the unit sphere $S^{2}$ (in $\mathbb{R}^{3}$ ) with respect to normalised surface measure $\mu$.

The aim of this note is to present another proof of Theorem 1. The proof is again computational in nature, but has the advantage over [4] in that it is based on a much more elementary and very well known functional equation. Namely, for a $(2 \times 2)$-matrix $D$ with distinct eigenvalues $\lambda_{1}$ and $\lambda_{2}$ and any $\mathbb{C}$-valued function $g$ defined on a subset of $\mathbb{C}$ containing $\sigma(D)=\left\{\lambda_{1}, \lambda_{2}\right\}$, the matrix $g(D)$ is given by the formula

$$
g(D)=\frac{g\left(\lambda_{1}\right)}{\left(\lambda_{1}-\lambda_{2}\right)} \cdot\left(D-\lambda_{2} I\right)-\frac{g\left(\lambda_{2}\right)}{\left(\lambda_{1}-\lambda_{2}\right)} \cdot\left(D-\lambda_{1} I\right)
$$

In particular, the proof given below provides an interesting and non-trivial application of (2).

To establish (i) $\Rightarrow$ (ii) is elementary and can be found in [4], for example. So let $A_{1}$ and $A_{2}$ be selfadjoint matrices in $L(\mathcal{H})$ which do not commute. To establish (ii) $\Rightarrow$ (i) it is to be shown that the distribution $T(A): \mathcal{S}\left(\mathbb{R}^{2}\right) \rightarrow L(\mathcal{H})$ has positive order. If $U$ is any orthogonal $(2 \times 2)$-matrix, define $U A U^{-1}=\left(U A_{1} U^{-1}, U A_{2} U^{-1}\right)$. Then $T\left(U A U^{-1}\right) f=U(T(A) f) U^{-1}$, for every $f \in \mathcal{S}\left(\mathbb{R}^{2}\right),[1$, Theorem $2.9(\mathrm{e})]$. So, choose for $U$ an orthogonal transformation such that the matrix $B_{1}$ of $U A_{1} U^{-1}$ with respect to the basis of $\mathcal{H}$ consisting of the orthonormal eigenvectors of $A_{1}$ is diagonal, say $\left(\begin{array}{cc}\alpha_{1} & 0 \\ 0 & \alpha_{2}\end{array}\right)$. Then the matrix $B_{2}$ of $U A_{2} U^{-1}$ with respect to this basis is of the form $\left(\begin{array}{cc}\beta_{1} & w \\ \bar{w} & \beta_{2}\end{array}\right)$ for some $w \in \mathbb{C}$ and $\beta_{1}, \beta_{2} \in \mathbb{R}$. Since $A_{1} A_{2} \neq A_{2} A_{1}$ it follows that $B_{1} B_{2} \neq B_{2} B_{1}$ and moreover, that $\alpha_{1} \neq \alpha_{2}$ (with $\alpha_{1}, \alpha_{2} \in \mathbb{R}$ ) and $w \neq 0$. Since the order of the distribution $T(B)$ is the same as that of $T(A)$ it suffices to show that $T(B)$ has positive order.

Fix $\xi=\left(\xi_{1}, \xi_{2}\right) \in \mathbb{R}^{2}$. For each $\lambda \in \mathbb{C}$, it follows that

$$
\begin{aligned}
\operatorname{det}(\lambda I-\langle\xi, B\rangle)=\lambda^{2}-\left(\xi_{1} \alpha_{2}+\xi_{2} \beta_{2}\right. & \left.+\xi_{1} \alpha_{1}+\xi_{2} \beta_{1}\right) \lambda \\
& +\left(\xi_{1} \alpha_{1}+\xi_{2} \beta_{1}\right) \cdot\left(\xi_{1} \alpha_{2}+\xi_{2} \beta_{2}\right)-|w|^{2} \xi_{2}^{2}
\end{aligned}
$$


Let $h=\left(\alpha_{1}-\alpha_{2}\right) / 2$ and $k=\left(\beta_{1}-\beta_{2}\right) / 2$, in which case $k \in \mathbb{R}$ and $h \in \mathbb{R} \backslash\{0\}$. Direct calculation shows that the solutions of $(3)$ are given by

$$
\lambda(\xi)=\frac{1}{2}\left(\xi_{1}\left[\alpha_{1}+\alpha_{2}\right]+\xi_{2}\left[\beta_{1}+\beta_{2}\right]\right) \pm\{\Delta(\xi)\}^{1 / 2},
$$

where $\Delta(\xi)=\left(h \xi_{1}+k \xi_{2}\right)^{2}+|w|^{2} \xi_{2}^{2}$. Since $\Delta(\xi)=0$ if and only if $\xi=0$, it follows from (4) that $\langle\xi, B\rangle$ has two distinct eigenvalues, say $\lambda_{1}(\xi)$ and $\lambda_{2}(\xi)$, whenever $\xi \neq 0$. The identity (2), with $D=\langle\xi, B\rangle$ and $g(z)=e^{i z}$, implies that

(5) $\quad e^{i\langle\xi, B\rangle}=\frac{e^{i \lambda_{1}(\xi)}}{\left(\lambda_{1}(\xi)-\lambda_{2}(\xi)\right)} \cdot\left(\langle\xi, B\rangle-\lambda_{2}(\xi) I\right)-\frac{e^{i \lambda_{2}(\xi)}}{\left(\lambda_{1}(\xi)-\lambda_{2}(\xi)\right)} \cdot\left(\langle\xi, B\rangle-\lambda_{1}(\xi) I\right)$,

for every $\xi \neq 0$. Of course, $e^{i\langle 0, B\rangle}=I$. Substituting (5) into (1), with $A$ replaced by $B$, shows that the $(1,2)$-entry of the matrix $T(B) f$ is given by

$$
L(f)=\frac{w}{2 \pi} \int_{\mathbb{R}^{2}} \frac{\left(e^{i \lambda_{1}(\xi)}-e^{i \lambda_{2}(\xi)}\right) \xi_{2} \hat{f}(\xi)}{\left(\lambda_{1}(\xi)-\lambda_{2}(\xi)\right)} d \xi, \quad f \in \mathcal{S}\left(\mathbb{R}^{2}\right) .
$$

If $\lambda_{1}(\xi)$ denotes the eigenvalue of $\langle\xi, B\rangle$ corresponding to the + sign in front of $\{\Delta(\xi)\}^{1 / 2}$ in (4), then it follows from (4) that (6) simplifies to

$$
L(f)=\frac{i w}{2 \pi} \int_{\mathbb{R}^{2}} \frac{\xi_{2} e^{i(\xi, u)} \hat{f}(\xi) \sin \{\Delta(\xi)\}^{1 / 2}}{\{\Delta(\xi)\}^{1 / 2}} d \xi, \quad f \in \mathcal{S}\left(\mathbb{R}^{2}\right),
$$

where $u=\left(\alpha_{1}+\alpha_{2}, \beta_{1}+\beta_{2}\right) / 2$. If $f_{u}(\eta)=f(u+\eta)$, for $\eta \in \mathbb{R}^{2}$, then $\widehat{f}_{u}(\xi)=$ $e^{i\langle\xi, u\rangle} \widehat{f}(\xi)$ and so

$$
L(f)=\frac{i w}{2 \pi} \int_{\mathbb{R}^{2}} \frac{\xi_{2} \widehat{f}_{u}(\xi) \sin \{\Delta(\xi)\}^{1 / 2}}{\{\Delta(\xi)\}^{1 / 2}} d \xi=\frac{w}{2 \pi} \int_{\mathbb{R}^{2}} \frac{\left(D_{2} f_{u}\right)(\xi) \sin \{\Delta(\xi)\}^{1 / 2}}{\{\Delta(\xi)\}^{1 / 2}} d \xi
$$

where $D_{2}$ denotes differentiation with respect to the second variable. By making the linear change of variables in $\mathbb{R}^{2}$ given by $y=M \xi$, where $M=\left(\begin{array}{cc}h & k \\ 0 & |w|\end{array}\right)$ and elements of $\mathbb{R}^{2}$ are interpreted as column vectors, it follows that

$$
L(f)=\frac{h|w| w}{2 \pi} \int_{\mathbb{R}^{2}} \frac{\left(D_{2} f_{u} \digamma\left(M^{-1} y\right) \sin \left(y_{1}^{2}+y_{2}^{2}\right)^{1 / 2}\right.}{\left(y_{1}^{2}+y_{2}^{2}\right)^{1 / 2}} d y, \quad f \in \mathcal{S}\left(\mathbb{R}^{2}\right) .
$$

The Fourier-Stieltjes transform $\widehat{\mu}$ of the measure $\mu$ (recall that $\operatorname{supp}(\mu)=S^{2} \subseteq$ $\mathbb{R}^{3}$ ) is easily computed via spherical polar coordinates and is given by

$$
\widehat{\mu}(\gamma)=\frac{\sin \left(\gamma_{1}^{2}+\gamma_{2}^{2}+\gamma_{3}^{2}\right)^{1 / 2}}{(2 \pi)^{3 / 2}\left(\gamma_{1}^{2}+\gamma_{2}^{2}+\gamma_{3}^{2}\right)^{1 / 2}}, \quad \gamma \in \mathbb{R}^{3} \backslash\{0\}
$$


with $\widehat{\mu}(0)=(2 \pi)^{-3 / 2}$. Let $\mathbb{D}$ be the closed unit disc in $\mathbb{R}^{2}$. Define a measure $\nu$ on the Borel subsets $\mathcal{B}\left(\mathbb{R}^{2}\right)$ of $\mathbb{R}^{2}$ by

$$
\nu(E)=\mu((E \cap \mathbb{D}) \times \mathbb{R}), \quad E \in \mathcal{B}\left(\mathbb{R}^{2}\right) .
$$

Given a function $\varphi: \mathbb{R}^{2} \rightarrow \mathbb{C}$, let $\tilde{\varphi}: \mathbb{R}^{3} \rightarrow \mathbb{C}$ be the function defined by $\tilde{\varphi}(x, y, z)=\varphi(x, y)$. A routine calculation shows that $\int_{\mathbb{R}^{2}} s d \nu=\int_{\mathbb{R}^{3}} \tilde{s} d \mu$, for every $\mathcal{B}\left(\mathbb{R}^{2}\right)$-simple function $s: \mathbb{R}^{2} \rightarrow \mathbb{C}$. It follows from the dominated convergence theorem that $\int_{\mathbb{R}^{2}} \varphi d \nu=\int_{\mathbb{R}^{3}} \tilde{\varphi} d \mu$ for every bounded Borel function $\varphi: \mathbb{R}^{2} \rightarrow \mathbb{C}$. In particular, putting $\varphi_{\xi}(x)=e^{i\langle\xi, x\rangle}$, for each fixed $\xi \in \mathbb{R}^{2}$, it follows that $\widehat{\nu}(\xi)=\widehat{\mu}(\xi, 0)$. That is,

$$
\widehat{\nu}(\xi)=\frac{\sin \left(\xi_{1}^{2}+\xi_{2}^{2}\right)^{1 / 2}}{(2 \pi)^{3 / 2}\left(\xi_{1}^{2}+\xi_{2}^{2}\right)^{1 / 2}}, \quad \xi \in \mathbb{R}^{2} \backslash\{0\},
$$

with $\widehat{\nu}(0)=(2 \pi)^{-3 / 2}$.

Now, the function $\Phi=\widehat{\nu}$ is locally integrable (as it is a continuous function vanishing at $\infty$ ) and hence, can be interpreted as a distribution in the usual way, that is, $\langle g, \Phi\rangle=\int_{\mathbb{R}^{2}} g(\xi) \Phi(\xi) d \xi$, for $g \in \mathcal{S}\left(\mathbb{R}^{2}\right)$. Accordingly, the distributional Fourier transform $\widehat{\Phi}$ of $\Phi$ is given by

$$
\langle p, \widehat{\Phi}\rangle=\langle\widehat{p}, \Phi\rangle=\int_{\mathbb{R}^{2}} \widehat{\nu}(\xi) \widehat{p}(\xi) d \xi=\int_{\mathbb{R}^{2}}\left(\int_{\mathbb{R}^{2}} e^{i(\xi, x)} d \nu(x)\right) \widehat{p}(\xi) d \xi
$$

for each $p \in \mathcal{S}\left(\mathbb{R}^{2}\right)$. Applying Fubini's theorem and the Fourier inversion formula $\int_{\mathbb{R}^{2}} e^{i\langle\xi, x\rangle} \widehat{p}(\xi) d \xi=2 \pi p(x)$ shows that

$$
\langle p, \widehat{\Phi}\rangle=2 \pi \int_{\mathbb{R}^{2}} p(x) d \nu(x), \quad p \in \mathcal{S}\left(\mathbb{R}^{2}\right) .
$$

Accordingly, the Fourier transform of $\Phi$ is the measure $2 \pi \nu$ (acting on $\mathcal{S}\left(\mathbb{R}^{2}\right)$ via the right-hand-side of (8)).

For $g \in \mathcal{S}\left(\mathbb{R}^{2}\right)$, let $g \circ M^{t} \in \mathcal{S}\left(\mathbb{R}^{2}\right)$ denote the function $x \mapsto g\left(M^{t} x\right)$, for each $x \in \mathbb{R}^{2}$, where $M^{t}$ is the transpose of the matrix $M$. Direct calculation shows that

$$
\left(D_{2} f_{u} \curlyvee\left(M^{-1} y\right)=\frac{1}{h|w|} \cdot\left(D_{2} f_{u} \circ M^{t}\right) \Upsilon y\right), \quad y \in \mathbb{R}^{2},
$$

for each $f \in \mathcal{S}\left(\mathbb{R}^{2}\right)$. It follows from (7), (8) and the definition of distributional Fourier transforms that

$$
L(f)=w(2 \pi)^{3 / 2} \int_{\mathbb{R}^{2}}\left(D_{2} f_{u} \circ M^{t}\right)(x) d \nu(x), \quad f \in \mathcal{S}\left(\mathbb{R}^{2}\right) .
$$


Since $f \mapsto f_{u}$ and $f \mapsto f \circ M^{t}$ are bicontinuous isomorphisms of $\mathcal{S}\left(\mathbb{R}^{2}\right)$ onto itself, it is clear from (9) that the distribution $L(f)$ has positive order. Since $L$ is the $(1,2)$-entry of $T(B) f$, for each $f \in \mathcal{S}\left(\mathbb{R}^{2}\right)$, it follows that $T(B)$ also has positive order.

The identity (9) shows that the support of $L$ is a translate of the image of $\mathbb{D}$ under a non-singular transformation in $\mathbb{R}^{2}$ (with positive determinant). In particular, $\operatorname{supp}(L)$ is an infinite subset of $\mathbb{R}^{2}$. Since $\operatorname{supp}(L) \subseteq \operatorname{supp}(T(B))=\operatorname{supp}(T(A))$ we have also given an alternative proof of the fact that $A_{1} A_{2}=A_{2} A_{1}$ if and only if $\operatorname{supp}(T(A))$ is a finite subset of $\mathbb{R}^{2},[4,5]$.

\section{REFERENCES}

[1] R.F.V. Anderson, 'The Weyl functional calculus', J. Funct. Anal. 4 (1969), 240-267.

[2] R.F.V. Anderson, 'On the Weyl functional calculus', J. Funct. Anal. 6 (1970), 110-115.

[3] P. Brenner, 'The Cauchy problem for symmetric hyperbolic systems in $L_{p}$ ', Math. Scand. 19 (1966), 27-37.

[4] B.R.F. Jefferies and W.J. Ricker, 'Commutativity for systems of $(2 \times 2)$ selfadjoint matrices', Linear and Multilinear Algebra 35 (1993), 107-114.

[5] W.J. Ricker, 'The Weyl calculus and commutativity for systems of selfadjoint matrices', Arch. Math. 61 (1993), 173-176.

[6] M.E. Taylor, 'Functions of several selfadjoint operators', Proc. Amer. Math. Soc. 19 (1968), 91-98.

[7] H. Weyl, The theory of groups and quantum mechanics (Dover Publ., New York, 1950).

School of Mathematics

The University of New South Wales

Sydney NSW 2052

Australia 